\title{
Structural, electronic and magnetic properties of vacancies in single-walled carbon nanotubes
}

\author{
W. Orellana and P. Fuentealba \\ Departamento de Física, Facultad de Ciencias, Universidad de Chile, Casilla 653, Santiago, Chile.
}

\begin{abstract}
The stability and properties of the monovacancy and the divacancy in single-walled carbon nanotubes (CNTs) are addressed by spin-density functional calculations. We study these defects in four nanotubes, the armchair $(6,6)$ and $(8,8)$ and the zigzag $(10,0)$ and $(14,0)$, which have diameters of about 8 and $11 \AA$, respectively. We also study different defect concentrations along the tube axis by increasing the supercell in this direction in order to have one defect every 13 and $26 \AA$ of CNT length. Our results show that in the equilibrium geometry CNTs with a monovacancy exhibit ferromagnetism with magnetic moments ranging from 0.3 to $0.8 \mu_{\mathrm{B}}$. On the other hand, CNTs with a divacancy do not exhibit magnetism due to the full reconstruction around the defect where all $\mathrm{C}$ atoms are three coordinated. We observe that the presence of a monovacancy does not change drastically the CNT electronic properties, preserving their corresponding metallic or semiconducting character. However, for the divacancy both armchair and zigzag CNT become semiconductors exhibiting a energy gap of about $0.15 \mathrm{eV}$.
\end{abstract}

\section{INTRODUCTION}

The study of radiation-induced defects in carbon nanostructures have become considerably important since the recent experimental results of roomtemperature magnetic ordering in proton-irradiated graphite, which exclude metal impurities $\frac{1}{\underline{1}}$, as well as the observation of ferromagnetism in pressure-induced polymerized fullerenes ${ }^{2.3}$. Magnetic ordering in defective graphite and fullerenes have been also predicted theoretically ${ }^{4.5}$, suggesting the possibility to realize nanoscale magnets with potential applications in spintronics and biocompatible magnetic materials. On the other hand, magnetism in single-walled carbon nanotubes (CNTs) appears to be more complex than graphite and fullerenes owing the curvature, chirality and different electronic characteristics of the tubes. Singlewalled CNTs, which are typically $10 \AA$ in diameter, can be metallic or semiconducting depending on the way that a graphene sheet is rolled up, which is characterized by the chiral indices $(n, m)$. Metallic tubes occur if $n-m$ is divisible by 3 , otherwise the tubes are semiconducting 6 . Tubes with indices $(n, 0)$ and $(n, n)$ are termed zigzag and armchair, respectively, which is related to the arrangement of carbon atoms around the tube.

Experimental knowledge concerning defect-induced magnetism in CNTs is still lacking. The difficult to measure magnetic ordering in nanotube samples resides in the presence of metal catalysts which are necessary to produce single-walled tubes. Theoretical investigations on open ended zigzag nanotubes are found to exhibit energetically favorable ferromagnetic spin configurations which are associated to unsaturated dangling bonds at zigzag edges ${ }^{5}$. Single vacancies in small-diameters CNTs, ranging from 4 to $8 \AA$, have been recently addressed by first-principles calculations ${ }^{7}$. Although relative high concentrations of vacancy or small supercell sizes containing a vacancy have been considered in this work, it shows that only metallic nanotubes with a single vacancy in their ground state equilibrium geometries would exhibit ferromagnetism.

\section{THEORETICAL APPROACH}

The calculations were performed in the framework of the spin-polarized density functional theory $\underline{\underline{8}}$, using a basis set of strictly-localized numerical pseudoatomic orbitals as implemented in the SIESTA code $\mathrm{e}^{\frac{9}{}}$. The exchange-correlation energy is calculated within the generalized gradient approximation ${ }^{10}$. Standard normconserving pseudopotentials ${ }^{11}$ in their separable form ${ }^{12}$ are used to describe the electron-ion interaction. We use a double- $\zeta$ singly-polarized basis set. We study the vacancies in both armchair and zigzag CNTs with different diameters. The armchair CNTs have chiral indices of $(6,6)$ and $(8,8)$, whereas the zigzag ones have indices of $(10,0)$ and $(14,0)$. For the $(6,6)$ and $(10,0)$ CNTs which have diameters of about $8 \AA$, we use unit cells containing 120 atoms. For the $(8,8)$ and $(14,0)$ CNTs with diameters of about $11 \AA$, the supercells contain 160 and 168 atoms, respectively. For both 8 and $11 \AA$ diameter CNTs the supercell has a length of $L \approx 13 \AA$. This means that we are calculating one defect every $13 \AA$ of CNT length. For the Brillouin zone sampling we use six $k$ points along the CNT axis $\stackrel{13}{~}$. To ensure negligible interaction between tubes we imposed a vacuum region of $12 \AA$. With the above supercells, the distance between vacancy images along the tube axis is about $13 \AA$. This means that we are simulating a CNT with a linear concentration of defects with one defect every $13 \AA$ of CNT length. A longer supercell with length of $2 L \approx 26 \AA$ are also consider to study the effect a lower defect density in the CNT electronic structure. To do that we use $(6,6)$ and $(10,0)$ nanotubes in supercell containing up to 240 atoms and the $\Gamma$ point for the BZ sampling. The positions of all atoms in the nanotubes were relaxed using the conjugated gradient algorithm until the force components become smaller 
(a)

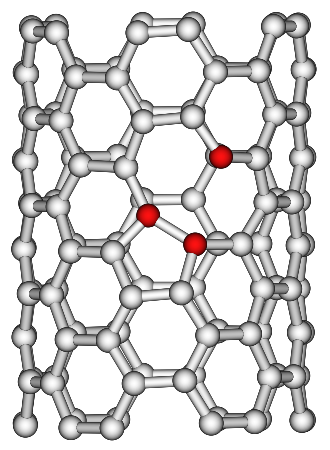

(c)

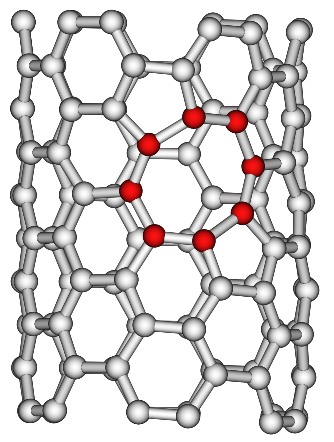

(b)

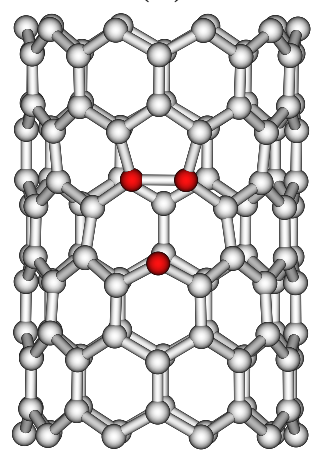

(d)

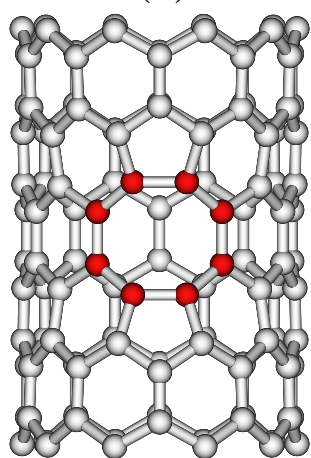

FIG. 1: Equilibrium geometry of the monovacancy and the divacancy in $(6,6)$ and $(10,0)$ CNTs. (a) $(6,6)+1 \mathrm{~V}$, (b) $(10,0)+1 \mathrm{~V}$, (c) $(6,6)+2 \mathrm{~V}$, (d) $(10,0)+2 \mathrm{~V}$. Painted balls display the $\mathrm{C}$ atoms around the defect.

than $0.05 \mathrm{eV} / \AA$.

\section{THE MONOVACANCY IN CARBON NANOTUBES}

Figures 1(a) and 1(b) show respectively the equilibrium geometries of the $(6,6)$ and $(10,0)$ CNTs containing the monovacancy (hereafter referred to as $(6,6)+1 \mathrm{~V}$ and $(10,0)+1 \mathrm{~V})$. We find that the neighboring $\mathrm{C}$ atoms partially reconstruct around the defect, forming a pentagon bonding structure, leaving a $\mathrm{C}$ atom twofold coordinated, which is projected slightly off to the nanotube surface. We also find metastable configurations for $(6,6)+1 \mathrm{~V}$ and $(10,0)+1 \mathrm{~V}, 10.5$ and $12.1 \mathrm{meV} /$ atom higher in energy that their respective ground state (GS) geometries. In these configurations the pentagon structure plus the twofold coordinated $\mathrm{C}$ atom are also found but rotated clockwise about $60^{\circ}$ with respect to the equilibrium geometries. A third metastable structure is found for $(10,0)+1 \mathrm{~V}$, which is $17.2 \mathrm{meV} /$ atom higher in energy than the GS geometry. Here, the $\mathrm{C}$ atoms neighboring to the vacancy do not form bond, remaining twofold coordinated. This geometry is known as $3 d b$ (three dangling bonds) structure.

Similar results for the GS and metastable geometries are found for the vacancy in the $11 \AA$ diameter CNTs $(8,8)$ and $(14,0)$. The metastable geometries of $(8,8)+1 \mathrm{~V}$ and $(14,0)+1 \mathrm{~V}$ are 7.3 and $6.7 \mathrm{meV} /$ atom higher in energy that the respective GS geometries. The smaller energy difference with respect to the $8 \AA$ diameter CNTs is attributed to a decrease in the strain energy due to the lower curvature of the $(14,0)$ and $(8,8)$ tubes. Thus, extrapolating to higher-diameter tubes, we can infer that the metastable geometries would tend to disappear. It is interesting to note that the $3 d b$ metastable structure of $(10,0)+1 \mathrm{~V}$ is not found in $(14,0)+1 \mathrm{~V}$, suggesting that the $3 d b$ geometry exits due to the higher strain in the $(10,0)$ tube, hindering further relaxations.

We calculate the formation energy of a vacancy in our CNTs using,

$$
E_{\text {form }}(V)=E_{t o t}(\mathrm{CNT}+\mathrm{V})-E_{t o t}(\mathrm{CNT})+\mu_{C}
$$

where $E_{t o t}(\mathrm{CNT}+\mathrm{V})$ and $E_{t o t}(\mathrm{CNT})$ are the total energy of the tube containing the vacancy and the perfect tube, respectively. $\mu_{C}$ is the carbon chemical potential which is calculated as the total energy per atom in the perfect tube. Our results are listed in Table I where we also include vacancy calculations performed in $(6,6)$ and $(10,0)$ CNTs considering a supercell with twice the length of the one previously used. We find that the vacancy in the $8 \AA$ diameter CNTs have similar formation energies, ranging from 5.65 to $5.85 \mathrm{eV}$, including the longer supercell. The metastable geometries also show the same tendency, with formation energies of about $7 \mathrm{eV}$. The above suggests a minor influence between images vacancies for supercell with lengths up to $L \approx 13 \AA$. For the vacancy in CNTs of $11 \AA$ of diameter we find lower formation energies than those of the $8 \AA$ ones, where the defective tube $(8,8)+1 \mathrm{~V}$ $[(14,0)+1 \mathrm{~V}]$ has a formation energy of about $1 \mathrm{eV}[0.5 \mathrm{eV}]$

TABLE I: Formation energy $\left(E_{\text {form }}\right)$ and magnetic moment $(m)$ for the monovacancy and the divacancy in armchair and zigzag CNTs, considering different supercell length $(L)$ or vacancy concentrations. $d_{\mathrm{C}-\mathrm{C}}$ is the equilibrium distance between $\mathrm{C}$ atoms that approach each other to form the pentagon bonding structure. In parenthesis are shown our results for the metastable geometries.

\begin{tabular}{lllll}
\hline \hline System & $L(\AA)$ & $m\left(\mu_{B}\right)$ & $E_{\text {form }}(\mathrm{eV})$ & $d_{\mathrm{C}-\mathrm{C}}(\AA)$ \\
\hline$(6,6)+1 \mathrm{~V}$ & 12.46 & $0.82(1.1)$ & $5.75(7.00)$ & $1.56(1.93)$ \\
$(6,6)+1 \mathrm{~V}$ & 24.92 & 0.63 & 5.85 & 1.57 \\
$(10,0)+1 \mathrm{~V}$ & 12.96 & $0.32(1.0)$ & $5.67(7.11)$ & $1.54(1.72)$ \\
$(10,0)+1 \mathrm{~V}$ & 25.92 & 0.49 & 5.65 & 1.53 \\
$(8,8)+1 \mathrm{~V}$ & 12.46 & $0.77(1.1)$ & $4.65(5.81)$ & $1.58(1.98)$ \\
$(14,0)+1 \mathrm{~V}$ & 12.96 & $0.53(1.1)$ & $5.20(6.32)$ & $1.58(1.74)$ \\
$(6,6)+2 \mathrm{~V}$ & 12.46 & $0.0(0.0)$ & $4.24(7.84)$ & $1.53(1.78)$ \\
$(6,6)+2 \mathrm{~V}$ & 24.92 & 0.0 & 4.17 & 1.52 \\
$(10,0)+2 \mathrm{~V}$ & 12.96 & $0.0(0.0)$ & $3.90(6.65)$ & $1.50(1.65)$ \\
$(10,0)+2 \mathrm{~V}$ & 25.92 & 0.0 & 4.06 & 1.49 \\
$(8,8)+2 \mathrm{~V}$ & 12.46 & 0.0 & 2.09 & 1.53 \\
$(14,0)+2 \mathrm{~V}$ & 12.96 & 0.0 & 2.33 & 1.50 \\
\hline \hline
\end{tabular}


(a)

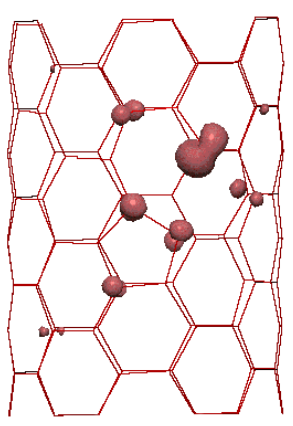

(b)

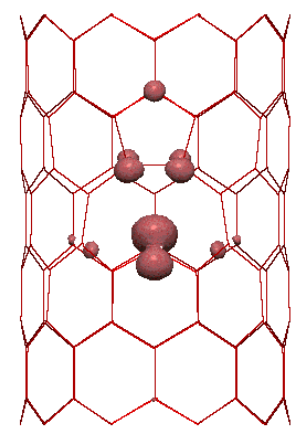

FIG. 2: Spin-density isosurfaces for the monovacancy in $8 \AA$ diameter CNTs. (a) $(6,6)+1 \mathrm{~V}$ and (b) $(10,0)+1 \mathrm{~V}$. The isosurfaces correspond to a charge of $0.015 \mathrm{e} / \AA^{3}$.

lower than that found for $(6,6)+1 \mathrm{~V}[(10,0)+1 \mathrm{~V}]$. The decrease in the formation energy is attributed to the decrease in the strain energy due to the small curvature of the larger tubes.

Table I shows our results for the magnetic moment of the defective CNTs. We find that all nanotubes under consideration exhibit ferromagnetic ordering. The lowerenergy structures of $(6,6)+1 \mathrm{~V}$ and $(10,0)+1 \mathrm{~V}$ have magnetic moments of 0.82 and $0.32 \mu_{\mathrm{B}}$, respectively, whereas for the metastable configuration the magnetic moment are closer to $1 \mu_{\mathrm{B}}$. Clearly, the magnetism is due to the under-coordinated $\mathrm{C}$ atom which has a localized unpaired spin, as shown in Fig. 2. However, as a $\mathrm{C}$ atom with a dangling bond has a magnetic moment of $1 \mu_{\mathrm{B}}$, the lower magnetic moments found in $(6,6)+1 \mathrm{~V}$ and $(10,0)+1 \mathrm{~V}$ are due to a redistribution of charge around the defect. This can be checked by looking at the equilibrium distance between the $\mathrm{C}$ atoms that form the pentagon structure, shown in Table I. As longer this distance is, larger is the magnetic moment. This means that once a vacancy is created, two of the three dangling bonds containing a spin rehybridize forming the pentagon structure. The weakness of this bond depend on the curvature strain of the tube. An extreme case occurs in the $3 d b$ configuration of $(10,0)+1 \mathrm{~V}$. Here no bond between the undercoordinated $\mathrm{C}$ atoms is formed, resulting in three dangling bonds with magnetic moment of $1.4 \mu_{\mathrm{B}}$. Considering lower concentration of vacancies, that is doubling the supercell length, we find that magnetic moments on $(6,6)+1 \mathrm{~V}$ and $(10,0)+1 \mathrm{~V}$ are 0.63 and $0.49 \mu_{\mathrm{B}}$, respectively. Thus, the magnetic moment tend to stabilize approximately around the average value $0.6 \mu_{\mathrm{B}}$ for more diluted monovacancy concentrations. The same behavior is observed for the tubes with higher diameters where the magnetic moment on $(8,8)+1 \mathrm{~V}$ and $(14,0)+1 \mathrm{~V}$ are 0.77 and $0.53 \mu_{\mathrm{B}}$, respectively. This stabilization appears to be related to a decrease in the strain energy on the tube surface. Thus, we may infer that the magnetization due to a single vacancy would be present in a wide variety of CNTs.
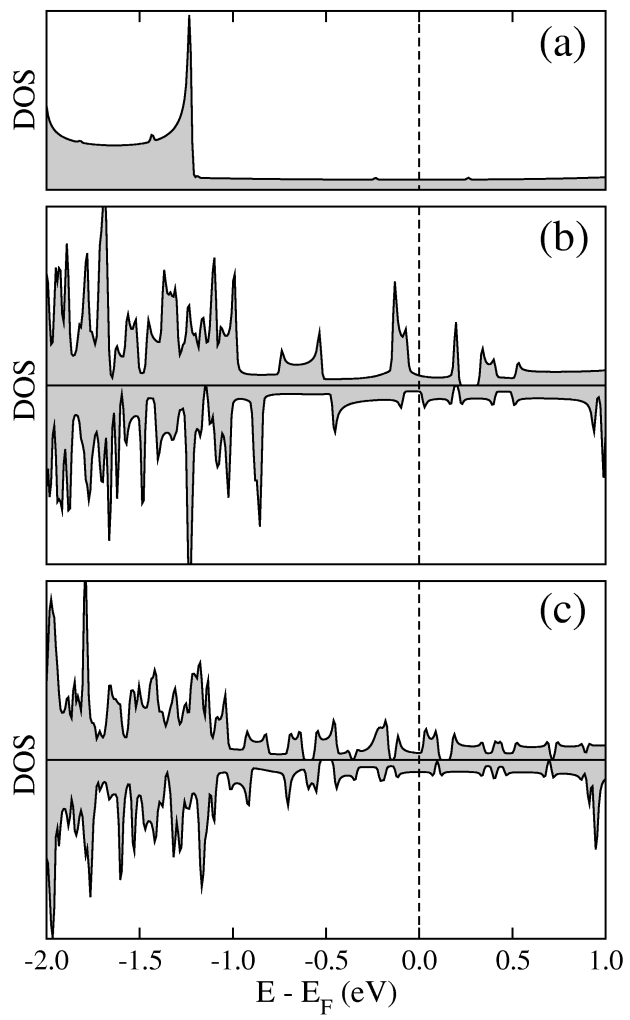

FIG. 3: Spin-resolved density of states (DOS) for the $(6,6)$ CNT with a monovacancy. (a) The perfect $(6,6)$ CNT (b) $(6,6)+1 \mathrm{~V}$ calculated with a supercell of $12.46 \AA$ length. (c) $(6,6)+1 \mathrm{~V}$ calculated with a supercell of $24.96 \AA$ length. In (a) and (b), upper and lower panels correspond to majority and minority spin configurations, respectively. Dashed lines indicate the position of the Fermi level $\left(\mathrm{E}_{\mathrm{F}}\right)$.

Figures 3(b) and 3(c) show the spin-resolved density of states for $(6,6)+1 \mathrm{~V} \mathrm{CNT}$ calculated with both supercells length (12.46 and $24.96 \AA$ respectively), which are compared with density of states of perfect $(6,6) \mathrm{CNT}$ [Fig. 3(a)]. We observe that the $(6,6)$ armchair CNT preserves its metallic character for different vacancy concentrations. We also note that the difference between majority (upper panel) and minority (lower panel) spin configurations in Fig. 3(b) and 3(c) clearly demonstrate the magnetism in the $(6,6)+1 \mathrm{~V}$ system. Similar results are found for $(8,8)+1 \mathrm{~V}$ CNT which suggests that these properties would be also found in larger diameter armchair CNTs. Figures 4(b) and 4(c) show the spin-resolved density of states for $(10,0)+1 \mathrm{~V}$ CNT calculated with supercells of 12.46 and $24.96 \AA$ length, and the density of states of perfect (10.0) CNT [Fig. 4(a)]. As we can see the $(10,0)$ semiconducting zigzag CNTs maintain this property when a $\mathrm{C}$ atoms is removed. For higher vacancy concentration the gap energy is about $0.1 \mathrm{eV}$ mainly due to a state located just above the Fermi energy. For lower vacancy concentration this state is less dispersive increasing the gap energy up to $0.2 \mathrm{eV}$. The presence of this states give to the $(10,0)+1 \mathrm{~V}$ CNT an acceptor character. 


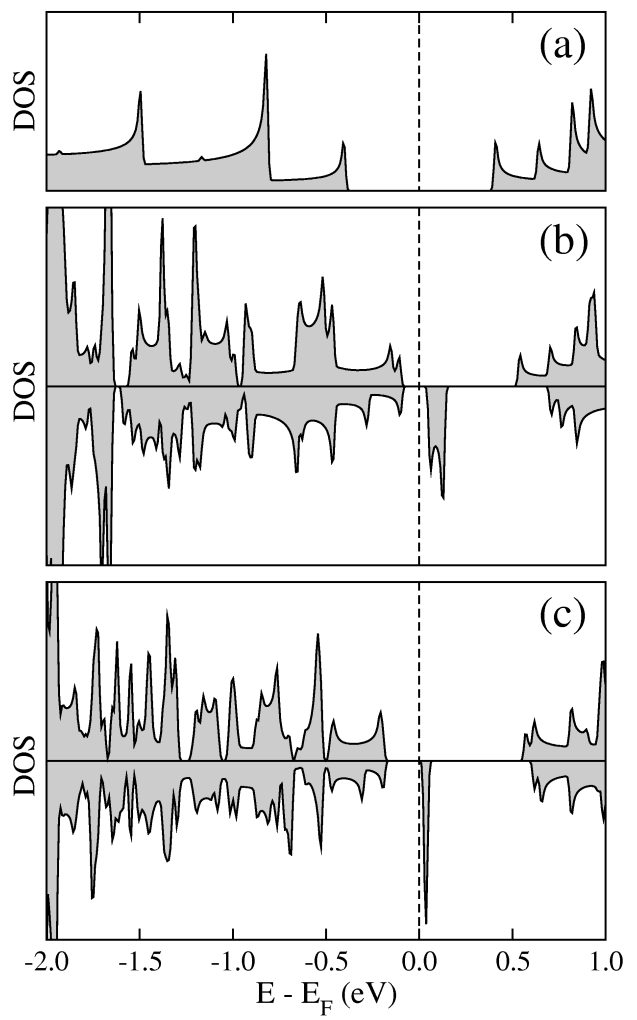

FIG. 4: Spin-resolved density of states for the (10,0) CNT with a monovacancy. (a) The perfect $(10,0)$ CNT (b) $(10,0)+1 \mathrm{~V}$ calculated with a supercell of $12.96 \AA$ length. (c) $(10,0)+1 \mathrm{~V}$ calculated with a supercell of $25.92 \AA$ length. Dashed lines indicate the position of the Fermi Level $\left(\mathrm{E}_{\mathrm{F}}\right)$.

\section{THE DIVACANCY IN CARBON NANOTUBES}

Figures 1(c) and 1(d) display the GS geometries for the divacancy in $(6,6)$ and $(10,0)$ CNTs (hereafter referred to as $(6,6)+2 \mathrm{~V}$ and $(10,0)+2 \mathrm{~V})$. We also find metastable geometries for $(6,6)+2 \mathrm{~V}$ and $(10,0)+2 \mathrm{~V}$, which are 10.5 and $23.3 \mathrm{meV}$ /atom higher in energy than the respective GS geometries. We observe that once a divacancy is created, the undercoordinated $\mathrm{C}$ atoms spontaneously reconstruct around the defect forming an octagon with two adjacent pentagon in opposite directions. For $(6,6)+2 \mathrm{~V}$ the pentagons point along a line of about $30^{\circ}$ with respect to the tube axis, whereas in the metastable structure the pentagons are aligned normal to the tube axis. For $(10,0)+2 \mathrm{~V}$ the pentagons align along the tube axis for the GS geometry and along a line of about $60^{\circ}$ with respect the tube axis. Similar reconstructed geometries for $(8,8)+2 \mathrm{~V}$ and $(14,0)+2 \mathrm{~V}$ CNTs where found, suggesting that these reconstructions should be found in larger diameters CNTs. For the $8 \AA(6,6)$ and $(10,0)$ CNTs the divacancy formation energies are found to be 4.2 and

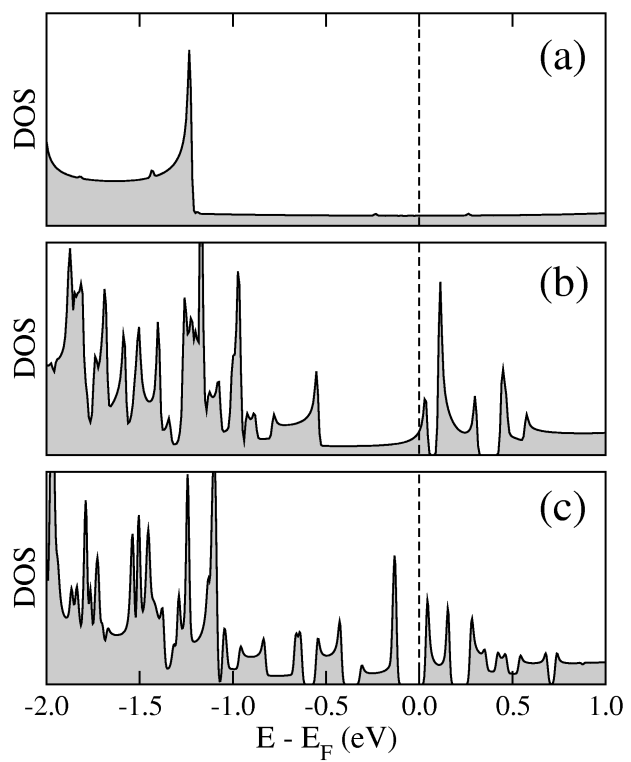

FIG. 5: Density of states for the $(6,6) \mathrm{CNT}$ with a divacancy. (a) Perfect $(6,6) \mathrm{CNT}$ (b) $(6,6)+2 \mathrm{~V}$ calculated with a supercell of $12.46 \AA$ length. (c) $(6,6)+2 \mathrm{~V}$ calculated with a supercell of $24.96 \AA$ length. Dashed lines indicate the position of the Fermi Level $\left(\mathrm{E}_{\mathrm{F}}\right)$.

$4.0 \mathrm{eV}$, respectively. Using longer supercell we note a small change in the formation energies suggesting a negligible interaction between the defect and their images in neighboring supercell. For the $11 \AA(8,8)$ and $(14,0)$ CNTs the formation energies decrease to 2.1 and $2.3 \mathrm{eV}$, respectively, about half the value found in the $8 \AA$ CNTs. This suggests that the curvature strain becomes important for the divacancy formation in small diameter CNTs. In Table I we also show the bond length between the $\mathrm{C}$ atoms that form the pentagon structure. The smaller bond length, of about $1.5 \AA$, are found for the divacancy in the zigzag $(10,0)$ and $(14,0)$ CNTs. As a consequence of the full reconstruction of the divacancy all $\mathrm{C}$ atoms around the octagon are threefold coordinated. Thus, no unpaired $\sigma$ electron is found, implying that carbon nanotubes with this defect do not exhibit magnetism. Therefore, we can infer that the origin of the magnetism in nanotubes and graphite are mainly due to unpaired $\sigma$ electrons which are localized. On the other hand, the delocalized $\pi$ electrons would have a negligible contribution because once an unpaired $\sigma$ electron reconstructs the magnetization disappear as shown our divacancy results.

Figures 5(b) and 5(c) shows the density of states for the $(6,6)+2 \mathrm{~V}$ CNT calculated with supercells of 12.46 and $24.96 \AA$ length, respectively, which are compared with the density of states of the perfect $(6,6)$ CNT [Fig. 5(a)]. We find that for the higher defect concentration the system has a metallic character with a small energy gap just above the Fermi energy, whereas for the lower concentration it become a semiconductor with a energy gap of about $0.15 \mathrm{eV}$. This behavior is originated in a dispersive CNT state which cross the Fermi level at about 


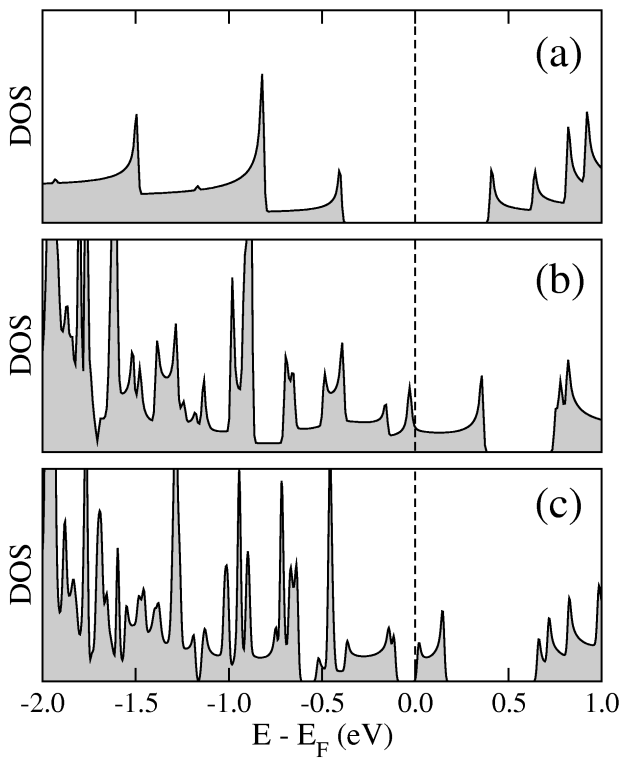

FIG. 6: Density of states for the $(10,0)$ CNT with a divacancy. (a) Perfect $(10,0)$ CNT (a) $(10,0)+2 \mathrm{~V}$ calculated with a supercell of 12.96 A length. (b) $(10,0)+2 \mathrm{~V}$ calculated with a supercell of $25.92 \AA$ length. Dashed line indicate the position of the Fermi Level $\left(\mathrm{E}_{\mathrm{F}}\right)$.

$0.8 \Gamma \mathrm{X}$. In the more diluted defect concentration this state becomes flat opening a energy gap and turning the system a semiconductor. Similar results are found for the $(10,0)+2 \mathrm{~V}$ CNT shown in Fig. 6 . Here, the perfect $(10,0)$ CNT is a semiconductor with an energy gap of $0.8 \mathrm{eV}$ [see Fig. 6(a)]. For the higher vacancy concentration [Fig. 6(b)] a defect state crosses the Fermi level at about $0.1 \Gamma \mathrm{X}$ while a CNT state rises close to the Fermi level, giving a metallic character to the system. However, for the more dilute defect concentration, the $(10,0)+2 \mathrm{~V} \mathrm{CNT}$ becomes semiconducting mainly due to the defect state above the Fermi level which becomes flat opening a energy gap of about $0.15 \mathrm{eV}$ as shown in Fig. 6(c). It is interesting to note that the $(8,8)$ and $(14,0)$ CNTs with a divacancy, calculated with supercells of $L \approx 13 \AA$, ex- hibit an energy gap of about $0.15 \mathrm{eV}$. This suggests that for more diluted defect concentrations in CNTs with diameters of at least $11 \AA$, the gap energy should increase due to the flattening of the defect states close to the Fermi energy.

\section{SUMMARY AND CONCLUSIONS}

We have studied the monovacancy and divacancy in armchair and zigzag CNTs with diameters of about 8 and $11 \AA$ and considering two concentrations of defects along the tube axis. Our results show that the CNTs with a monovacancy exhibits a ferromagnetic ordering induced by an undercoordinated $\mathrm{C}$ atom. The magnetic moment in the defect equilibrium geometries fluctuate among 0.3 and $0.8 \mu_{\mathrm{B}}$, depending on the defect concentration and on the diameter of the CNTs. The electronic density of states along the CNTs axis show that the monovacancy does not change the electronic character of the tubes. On the other hand, once a divacancy is created the neighboring $\mathrm{C}$ atoms spontaneously reconstruct around the defect forming an octagon with two adjacent pentagon in opposite directions. Because all the $\mathrm{C}$ atoms are three coordinated in this defect, CNTs with a divacancy are not magnetic. However, the electronic properties of CNTs change when they contain a divacancy where both armchair and zigzag CNTs become semiconductor having a energy gap of about $0.15 \mathrm{eV}$. The above properties suggest the possibility to achieve nanoscale magnets and magnetic patterns with potential applications in spintronics and biocompatible magnetic materials.

\section{Acknowledgments}

We thank Millennium Nucleus of Applied Quantum Mechanics and Computational Chemistry for financial support, under project P02-004-F. WO also thanks FONDECYT for partially support this work under project No. 1050197.
1 P. Esquinazi, D. Spemann, R. Höhne, A. Setzer, K.-H. Han, and T. Butz, Phys. Rev. Lett. 91 (2004) 227201.

2 T. Makarova, B. Sundqvist, P. Esquinazi, et al., Nature 413 (2001) 718.

3 R.A. Wood, M.H. Lewis, M.R. Müller, D. Eckert, et al., J. Phys.: Condens. Matter 14 (2002) L385.

4 P.O. Lehtinen, A.S. Foster, Y. Ma, A.V. Krasheninnikov, and R.M. Nieminen, Phys. Rev. Lett. 93 (2004) 187202.

5 Y.-H. Kim, J. Choi, K.J. Chang, and D. Tománek, Phys. Rev. B 68 (2002) 125420.

${ }^{6}$ M.S. Dresselhaus, G. Dresselhaus, and Ph. Avouris, Carbon nanotubes: Synthesis, Structure, Properties and Applications (Springer-Verlag, New York, 2001).

7 Y. Ma, P.O. Lehtinen, A.S. Foster, and R.M. Nieminen,
New J. Phys. 6 (2004) 68.

${ }^{8}$ W. Kohn, Rev. Mod. Phys. 71 (1999) 1253.

9 P. Ordejón, E. Artacho, and J. M. Soler, Phys. Rev. B 53 (1996) R10441; J.M. Soler, E. Artacho, J.D. Gale, A. García, J. Junquera, P. Ordejón, D. Sánchez-Portal, J. Phys.: Condens. Matter 14 (2002) 2745.

10 J.P. Perdew, K. Burke, and M. Ernzerhof, Phys. Rev. Lett. 77 (1996) 3865.

11 N. Troullier and J. L. Martins, Phys. Rev. B 43 (1991) 1993.

12 L. Kleinman and D.M. Bylander, Phys. Rev. Lett. 48 (1982) 1425.

13 H.J. Monkhorst and J.D. Pack, Phys. Rev. B 13 (1976) 5188. 